\title{
The Value of High Antistreptolysin 0 Titre as an Indicator of Tonsillectomy in Upper Egypt
}

\author{
Essam A. Abo El-magd1, Mona Abdel Meguid', Abd El Rahman El Tahan' \\ ${ }^{1}$ Department of Otolaryngology, Faculty of Medicine, Aswan University, Egypt \\ ${ }^{2}$ Department of Clinical Pathology, Faculty of Medicine, Al Azhar University, Asuit, Egypt \\ Email: esamali801@yahoo.com
}

Received 25 July 2015; accepted 12 January 2016; published 15 January 2016

Copyright (C) 2016 by authors and Scientific Research Publishing Inc.

This work is licensed under the Creative Commons Attribution International License (CC BY). http://creativecommons.org/licenses/by/4.0/

(c) (i) Open Access

\begin{abstract}
Background: In this study, we aimed to evaluate the benefit of performing of tonsillectomy in patients with raised serum ASO titre only in absence or presence of group A beta heamolytic streptococci (GABHS) in throat swab. Materials and methods: In this prospective cohort study, 196 patients below the age of 14 were suffering from non-specific streptococcal infections, without fulfilling the clinical parameters used for the diagnosis of chronic tonsillitis 156 patients had a raised ASO titre above $200 \mathrm{iu} / \mathrm{ml}$. Throat swab culture was performed in all patients. Results: The results showed that out of the 156 patients, 52 had positive throat swab for GABHS. All of the patients underwent tonsillectomy. Follow-up of the patients for one year after the operation, $88 \%$ of the first group with high ASO titre and positive throat swab showed improvement of symptoms; $25 \%$ of the second group with high ASO titre and negative throat swab showed improvement of symptoms. Conclusion: Our study shows that isolation of GABHS from the patients tonsils by throat swab along with high ASO titre may be an indication of tonsillectomy in absence of any other indications.
\end{abstract}

\section{Keywords}

GABHS, Non Specific Strept Infection, ASO Titre, Throat Swab Culture, Tonsillectomy

\section{Introduction}

A raised ASO titre level is one of the most relevant retrospective serological indices of antecedent GABHS infection. A single titre of more than $200 \mathrm{iu} / \mathrm{ml}$ is considered as a raised value. Serum ASO titre is raised when 
there is an infection of any organ of the body by GABHS, so the increased serum ASO titre should not be the only deciding criterion for tonsillectomy if GABHS is not present in the palatine tonsils [1]. GABHS is the most common bacteria that cause acute tonsillitis. Streptococcal infection can lead to rheumatic fever. The incidence of rheumatic fever in untreated cases of tonsillitis is $3 \%$ and in treated cases, the incidence falls to $0.3 \%$. It has been estimated that rheumatic heart disease constitutes $25 \%$ to $40 \%$ of all cardiovascular diseases in third world countries [2]. Recurrent tonsillitis is the most common indication of tonsillectomy. Recurrent infections are further defined as [3]:

1) 7 or more attaches for one year or;

2) 5 attaches per year for 2 years or;

3) 3 attaches per year for 3 years.

Clinical parameters used for the diagnosis of chronic tonsillitis were as follows [2]:

1. Recurrent attaches of tonsillitis;

2. Tender, atrophic or hypertrophic tonsils with persistent sore throat;

3. Congested anterior pillar;

4. Palpable jugulodigastric lymph node;

5. Expression of cheesy material from crypts on applying pressure on the tonsils.

In cases of infrequent recurrent tonsillitis and sings of chronic tonsillitis cannot be detected, it is difficult to take the decision of tonsillectomy. Patients complaining of non-specific manifestations such as anorexia and failure to thrive, myalgia and arthralgia, recurrent upper respiratory tract infection and recurrent otitis media may get benfite from tonsillectomy. Colonization of the tonsils by GABHS is also an important indication of tonsillectomy. To establish the carrier state of GABHS in tonsils, different investigations have been advocated the following [1]:

1. Throat swab culture;

2. Fine needle aspiration from the core of the tonsil;

3. Serum antistreptlysin $\mathrm{O}$ titre;

4. Core culture of the dissected tonsil after tonsillectomy.

\section{Materials and Methods}

We designed a prospective cohort study, recruiting 196 children suffering from repeated sore throat and upper respiratory tract infection, post-streptococcl arthralgia and arthritis (arthritis after pharyngeal infection with beta hemolytic streptococcal in patients had no other criteria of rheumatic fever) and any other non specific strept infection manifestation. The study was conducted in the ENT department of Qena university hospital, south valley university. Egypt from January 2012 to December 2012. One hundred and fifty six patients who showed raised ASO titre above $200 \mathrm{iu} / \mathrm{ml}$ were included in this study. Throat swab were cultured from pyogenic organisms from the surface of the tonsil using sterile swab sticks. Then the swab was immediately inoculated into nutrient agar, blood agar and McConkeys agar plates. Identification of the bacteria was done after isolation as per the standard procedure. A detailed history of each patient was taken which include the chief complaint, history of present illness and past illness. The onset, duration and progress of the symptoms were taken. After complete clinical examination, blood and urine examination were done. Blood examination included complete blood picture, bleeding time, prothrombin time and concentration, partial thromboplastin, ESR and CRP. Urine was examined for sugar and albumen. All the patients underwent tonsillectomy under general anesthesia by dissection method. Consent for all these procedures were obtained from the guardians. Patients who had an acute tonsillitis, peritonsillar abscess or suspected neoplasm were excluded from this study. Follow up the patient-for one year as regard clinical improvement of symptoms. Statistical analysis was done using Chi square method.

\section{The Results}

This study consisted of 85 female and 71 male. The youngest patient was 4 years and the oldest was 14 . From those 156 patients 52 patients showed GABHS in throat swab culture while 104 patients had negative throat swab culture.

Of the 104 patients with elevated ASO titre and negative swab for GABHS:

1. 60 patients were complaining of recurrent sore throat and 18 patients improved after tonsillectomy (30\%);

2. 44 patients were complaining of post streptococcal arthritis and 11 patients improved after tonsillectomy 
(25\%).

Of the 52 patients with elevated ASO titre and positive throat swab for GABHS:

1. 30 patients had recurrent sore throat, of those patients 26 were improved after tonsillectomy (86.6\%);

2. 22 patients had recurrent post streptococcal arthritis, 18 patients were improved after tonsillectomy (81.8\%)

As regard to major complaint of the study participants in Table 1 , the results showed repeated sore throat found in 55 patients, while other complaints like anorexia and failure to thrive and repeated upper respiratory tract infections present only in 30 patients.

According to number of episodes of sore throat after tonsillectomy during one year follow-up in the first group, Table 2 showed that, more than 2/3 of patients (70\%) have three or more attacks per year.

On the other hand there are 3 or more attacks of post streptoccal arthritis after tonsillectomy in $3 / 4$ (75\%) of patients (Table 3).

Regarding number of attacks of sore throat and arthritis after tonsillectomy in the second group with positive throat swab, the majority of them $(\mathbf{8 6 . 6 \% )}$ ) and $(\mathbf{8 1 . 8 \% )}$ ) have less than 3 attacks per year respectively (Table 4 and Table 5).

According the result showed in Table 6, the majority of patients (84.6\%) of group B versus (27.8\%) of patients of group A improved after tonsillectomy.

\section{Discussion}

A high ASO titre indicates the presence of a recent streptococcal infection [4]. A rise of ASO antibody generally takes place from one to four weeks after streptoccal infection and after that the titre returns to the previous level

Table 1. Characteristics of 196 patients.

\begin{tabular}{cc}
\hline Number of patients (196) & Complaint \\
\hline 55 patients & Repeated sore throat \\
46 patients & Non specific arthragia and myalgia \\
35 patients & Repeated otitis media \\
30 patients & Anorexia and failure to thrive \\
30 patients & Repeated upper respiratory tract infection \\
\hline
\end{tabular}

Table 2. Number of episodes of sore throat after tonsillectomy during one year follow up in the first group.

\begin{tabular}{ccc}
\hline Number of attacks of sore throat & Number of patients & Percentage \\
\hline $\begin{array}{c}\text { >three attacks per year } \\
\text { <three attacks }\end{array}$ & 42 & $70 \%$ \\
\hline
\end{tabular}

Table 3. Number of attacks of post streptoccal arthritis after tonsillectomy.

\begin{tabular}{ccc}
\hline Number of attacks after 1 year & Number of patients & Percentage \\
\hline$>$ three attacks per year & 33 & $75 \%$ \\
$<$ three attacks per year & 11 & $25 \%$ \\
\hline
\end{tabular}

Table 4. Number of attacks of sore throat after tonsillectomy in the second group with positive throat swab.

\begin{tabular}{ccc}
\hline Number of attacks of sore throat & Number of patients & Percentage \\
\hline >three attacks per year & 4 & $13.4 \%$ \\
<three attacks per year & 26 & $86.6 \%$ \\
\hline
\end{tabular}


Table 5. Number of attacks of post streptococcal arthritis.

\begin{tabular}{ccc}
\hline Number of attacks of arthritis & Number of patients & Percentage \\
\hline three attacks per year & 4 & $18.2 \%$ \\
<three attacks per year & 18 & $81.8 \%$ \\
\hline
\end{tabular}

Table 6. Comparison between the group with high titre and negative throat swab (group A) versus the second group with high titre and positive throat swab (group B).

\begin{tabular}{ccc}
\hline Groups & Number of improved cases & Percentage \\
\hline Group A 104 patients & 29 & $27.8 \%$ \\
Group B 52 patients & 44 & $84.6 \%$ \\
\hline
\end{tabular}

$(\mathrm{p}<0.001)$.

within two or three months. Throat swab culture is positive in $80 \%$ of streptococcal infections but may be negative in cases of chronic infection. Antigen detection test is very sensitive, but it is very costly and not available in all the centers. ASOT titre test is the most widely used test. It is more popular because of its availability in our country, less cost and reasonable sensitivity. Very few studies have been reported regarding the value of ASO tire as an indicator of tonsillectomy. So, comparative discussion is limited. Indication of tonsillectomy depends mainly on clinical diagnosis. History of five or more episode of tonsillitis per year for at least one year, disabling episodes of sore throat prevent normal functions and sore throat due to tonsillitis [1] On examination 1) tender, atrophic or hypertrophic tonsils with persistent sore throat; 2) presence of congested anterior pillar; 3) palpable jugulodigastric lymph node; 4) expression of cheesy material from crypts on applying pressure on the tonsils [2]. In our study these clinical finding were not conclusive but the patients were complaining and the tonsils were accused. Of 104 patients with high ASO titre above $200 \mathrm{iu} / \mathrm{ml}$ and negative throat swab, 60 patients had recurrent sore throat more than three times per year which were disabling episodes with bad effect on normal daily life. After tonsillectomy only 18 patients improved with percentage of $30 \%$. The remaining 44 patients had recurrent attacks of arthritis only 11 patients improved after tonsillectomy with a percentage of $25 \%$. Reactive arthritis has been defined as a sterile inflammatory arthritis occurring in conjunction with bacterial infection at a site distant from the joints. Previous reports have postulated the relationship between streptococcal tonsillitis and reactive arthritis. It has thus been termed post-streptococcal reactive arthritis rather than acute rheumatic fever [5]. 52 patients with high ASO titre and positive throat swab, 30 patients had recurrent sore throat and 26 patients improved after tonsillectomy with a percentage of $86.6 \%$. The remaining 22 patients had recurrent attacks of arthritis, 18 patients improved after tonsillectomy with a percentage of 81.8. In comparison between the first group with high ASO titr and negative throat swab (104) and the second group with high ASO titre and positive swab (52), the improved rate was $27.4 \%$ in the first group and $84.4 \%$ in the second group with a p value of 0.001 which is statistically significant. Our results are in line with Hombrom et al. 2012 [1] who concluded that one should perform throat swab culture along with ASO titre before doing tonsillectomy in absence of any other indications. Viswanathan et al. 2000 [2], reported that there were significant reduction of sore throat attacks after tonsillectomy within one year after operation. They concluded that tonsillectomy had a significant role in preventing or reducing recurrent streptococcal throat infection. This observation is very important, since incidence of rheumatic fever and rheumatic reactivation can be reduced by prevention of streptococcal infection. Motanoski et al. [6] showed that patients who had under gone surgery have lower infection rate with GABHS. Paradise et al. [7] also found that throat infection was markedly reduced in the first 2 years after tonsillectomy. Kobayashi et al. [8] suggested that a reactive arthritis induced by tonsillitis and cured by tonsillectomy. Other studies investigated the link between arthritis and tonsillectomy. The effect of tonsillectomy on rheumatoid arthritis was reported as a decrease in the degree of pain and amelioration of the disease [9] [10].

\section{Conclusion}

From the results of this study, it is evident that one should perform throat swab culture with ASO titre before doing tonsillectomy in absence of any other indications. A high ASO titre only is not an indicator of tonsillect- 
omy. A high ASO titre with positive throat swab culture can be considered as an indication of tonsillectomy.

\section{References}

[1] Hembrom, R., Roychaudhuri, B.K. and Saha, A.K. (2012) Evaluation of the Validity of High ASOT only as an Indicator of Tonsillectomy. Indian Journal of Otolaryngology and Head \& Neck Surgery.

[2] Viswanathan, N. and Sasikumaran, S. (2000) Effect of Tonsillectomy on ASO-Titre. Indian Journal of Otolaryngology and Head \& Neck Surgery, 52, 329-331.

[3] Dhingra, P.L. (2010) Diseases of Ear Nose and Throat. Fourth Edition, Elsevier, India, 382.

[4] Satoshi, F., Hanwa, Y., et al. (1988) Streptococcal Antibody: As an Indicator of Tonsillectomy. Acta Oto-Laryngologica, 454, 286-291.

[5] Deighton, C. (1993) Beta Haemolytic Streptococci and Reactive Arthritis in Adults. Annals of the Rheumatic Diseases, 52, 475-482. http://dx.doi.org/10.1136/ard.52.6.475

[6] Motonoski, G.M., et al. (1968) Epidemiology of Streptococcal Infections in Rheumatic and Non Rheumatic Families. American Journal of Epidemiology, 87, 226.

[7] Paradise, J.L., Bluestone, C.D., Bachman, R.Z., et al. (1984) Efficacy of Tonsillectomy for Recurrent Throat Infection in Severly Affected Children; Results of Parallel and Non Randomized Clinical Trials. The New England Journal of Medicine, 310, 674-683. http://dx.doi.org/10.1056/NEJM198403153101102

[8] Kobayashi, S., Tamura, N., Akimoto, T., et al. (1996) Reactive Arthritis Induced by Tonsillitis. Acta Oto-Laryngologica, 523, 206-211.

[9] Kataura, A. and Tsubota, H. (1996) Clinical Analysis of Focus Tonsil and Related Diseases in Japan. Acta Oto-Laryngologica, 523, 161-164.

[10] Kawano, M., Okada, K., et al. (2003) Simultaneous, Clonally Identical T Cell Expansion in Tonsil and Synovium in Patients with Rheumatoid Arthritis and Chronic Tonsillitis. Arthritis \& Rheumatism, 48, 2483-2488.

http://dx.doi.org/10.1002/art.11212 